\title{
Secondary Traumatic Stress and Resilience in Intensive Care Doctors and Nurses
}

\author{
Ceberio MR*1, Jones GM${ }^{1}$, Benedicto $\mathrm{G}^{1}$ and Aizpurua $\mathrm{RI}^{2}$ \\ ${ }^{1}$ Neuroscience and Social Sciences Research Laboratory (LINCS), University of Flores (UFLO), \\ Argentina \\ ${ }^{2}$ Department of Psychotherapy, Queensland Program of Assistance to Survivors of Torture and \\ Trauma, Australia
}

*Corresponding author: Marcelo Rodriguez Ceberio. Neuroscience and Social Sciences

\section{Research Article}

Volume 5 Issue 2

Received Date: June 14, 2021

Published Date: August 31, 2021

DOI: $10.23880 / \mathrm{mhrij}-16000147$

Research Laboratory. University of Flores (UFLO), Argentina, Tel: 54 91154180231; Email: marceloceberio@gmail.com

\section{Abstract}

The Intensive Therapy Unit (ICU) is a hospital or clinic service that has certain peculiarities. Al- though, the work in the different specialties is covered with stress, the ICU is the place where the emotional impacts, the speed of the interventions, the severity of the pathologies, the complexity of the medical equipment and its domain, are some of the factors that generate greater stress. The present research explores through different instruments, explores vulnerability through the correlation between the impact of STSs and resilient actions that would simultaneously act as a buffer for the emotional shocks that appear in daily work in medical and nursing personnel. At the same time, it investigates the traumatic impact of children and the current one on ICU services.

Keywords: Vulnerability; Resilience; Traumatic Impact; Life; Intensive Therapy

Abbreviations: STS: Secondary Traumatic Stress; ICU: Intensive Care Unit; DSM: Diagnostic and Statistical Manual of Mental Disorders.

\section{Introduction: between Vulnerability and Resilience}

The "intensive therapy" or the "intensive care unit" is the epicenter of the hospital space where the struggle between life and death is most observed. The patients who are at higher risk of dying, the people who need more help due to the severity of their pathology, are hospitalized in that place $[1,2]$. The care environment in an Intensive Care Unit is considered a highly complex environment, since it requires highly professional medical personnel and state-of-the- art equipment. It is a context that presents an accelerated rhythm, high anxiety levels in which aggressive and invasive procedures are performed, and where grief is permanently debated, with death, many times, being one of the results [2]. These emergency situations lead to triggering high levels of stress, anxiety, anguish, annoyance, irritability and other emotions that are associated with stress [3]. Secondary traumatic stress (STS) is a syndrome present mainly in health professionals who work with people in distress [4].

The STS is also known as "empathy burnout", that is, empathy is the ability to place oneself in the place of the other, to achieve personally experience what the other feels or thinks [5]. From this professional position results the stress generated by the natural tendency to want to help people in 


\section{Mental Health \& Human Resilience International Journal}

suffering, producing a reduction in the ability and interest to deal with the suffering of others [6]. It is a response to stress that emerges suddenly and includes a feeling of helplessness, loneliness and confusion. If not diagnosed and cared for in due time, this can lead to depression and other stress-related illnesses [7]. The process that generates this pathology is complex, since it includes organizational and contextual variables, as well as individual reactions, vulnerability and resilience, which exert an important influence. Vulnerability can be defined as the diminished ability of a person or a group of people to anticipate cope with and resist the effects of a hazard produced by nature or caused by human activity; while the possibility or ability to recover refers to resilience. Situations of natural disasters $[8,9]$ such as earthquakes, tidal waves, tsunamis, volcanic eruptions, forest fires, or events caused by humans such as murders, criminal acts, sexual abuse, rape, violence of all kinds, dictatorships, Economic hecatombs, political crises, etc., constitute part of the list of events that can disturb the stability of people [10].

Therefore, then, there are two types of human vulnerability: an anthropological vulnerability, understood as a condition of intrinsic fragility of the human being as a biological being, and a sociopolitical vulnerability, understood as belonging to a social group that implies economic, cultural, and social factors. Environmental and political issues that make human beings vulnerable [11]. But these definitions associate factors of the general context that violate the lives of the people who make it up. But there are situations that are not general, but specific, as in this case of the intensive care environment, where vulnerability is produced by factors of the activity carried out that operates as a stressor and generates wear on the limbs involved.

However, vulnerability is subjective and this is equivalent to saying that each person will build their own vulnerability according to the attribution of meaning granted to the fact [12]. A critical event that happens in a context will not be the same event that makes everyone vulnerable. It is not a traumatic event in itself, but will depend on the attribution of meaning that each person in that context gives it. Each person will make his particular crisis out of the general crisis. There are as many versions of the critical situation as there are people who suffer from it. Therefore, vulnerability is subjective. Environmental impact is impact in terms of the meaning attributed to the event $[13,14]$.

But in the face of this situation that generates vulnerability, resilience has significant relevance in the present study, since it represents the human capacity to flexibly assume limit situations and overcome them $[15,16]$, so it is considers it as a protective factor. They are the resources available to develop effective strategies in times of crisis. Resilience is the ability to live and develop positively despite the traumatic episodes that may have occurred. It is a concept derived from physics and chemistry, and describes the ability of steel to recover its initial shape de- spite the shocks and use it may receive and the efforts that may be made to deform it. It is a term that is taken from the resistance of materials that bend without breaking to recover the original situation or shape [17].

If a person manages to overcome the critical situation, he comes out of it strengthened. He means that the overcome experience translates into personal value, strength, security to face future situations. Resilience is a great source of feedback. Neuroscience affirms that the most resilient individuals have greater emotional balance in the face of stressful situations, cushioning the pressures, with which, the control in front of the experiences and greater capacity to face the facts is increased. In stressful situations, that capacity for resistance and resilience is put into play [13]. Many situations in a person's life have these characteristics, for example: rapes, sexual abuse, physical violence and abuse, the unexpected death of a loved one, terminal illnesses, emotional abandonment, separations, failure, natural disasters, wars, political persecutions, extreme poverty, etc. Al- though there are people who have a natural tendency to confront difficult situations and oppose their own capacities, that is, a resilient attitude, and resilience is learned. The awareness of our personal values, of our possibilities, being self-aware of what we can and what we are not capable of, is a way of exercising resilience $[15,16]$. It is the notion of empowerment. And the psychotherapy space is a resilient learning place, like so many human relationships. The resilient attitude is the behavioral tendency of certain people in the face of adverse situations. It is a confluence of unconscious, interactional, cognitive, emotional, biochemical, neurobiological epigenetic factors [18] that come together in a resilient attitude towards life that tries to overcome the traumatic situation.

\section{Intensive therapy and crisis}

Everything in the context of an Intensive Care Unit (ICU) is a constant crisis. Crises are defined as states of maximum tension that tend to destabilize people balance [19]. There are expected crises in life such as births, deaths, moves, trips, etc.; but there are others outside the script of life such as deaths of young people, unexpected terminal illnesses or accidents, among others. They all generate changes; they are situations that from instability cause a new stability [20]. The ICU is a special facility within the hospital or clinic where patients who are in a serious situation to the point of being at risk of life are treated, therefore requiring monitoring of their vital signs and other parameters. Attention is minute by minute. 


\section{Mental Health \& Human Resilience International Journal}

In this Unit there are a multiplicity of stress-generating situations, for example: the fight against time, since frequently doctors and nurses must make decisive decisions for the patient's life in a climate of precipitation [21]; the risk of malpractice; rotating shifts; the suffering and death of patients (often children and young people); lack of supply of medication; handling of highly complex equipment, etc. All these factors complicate the situation of the good development of emergency care [22]. At this point, it is important to reflect on the risks in the psychophysical health and, consequently, also in the quality of life of these professionals since there are numerous negative consequences that the possibility of suffering secondary traumatic stress brings with it. These consequences include: increased levels of depression [23] and increased risk of suicide [24].

With the possibility of carrying out a proper awareness and prevention, situations such as: the in- crease in medical leave, and absenteeism from work, mainly due to "Burn Out" and work stress, etc. would be avoided [25]. These factors decrease the effectiveness of physicians to the detriment of patients [26-28]. In the present investigation, STS is taken as a form of Post-Traumatic Stress Disorder, since the diagnostic criteria of the fifth edition of the Diagnostic and Statistical Manual of Mental Disorders (DSM-5), clarify that secondary exposure can lead to Symptoms belonging to this disorder in which three dimensions are observed: reexperimentation, avoidance and hyper vigilance. This work aims to analyze the presence of STS symptoms and levels of resilience in intensive care doctors and nurses in Argentina, and then analyze the relationship between both variables. The possibility of showing an important correlation between low levels of STS symptoms and high levels of resilience, would allow us to advance in raising awareness about the importance of promoting the development of resilience and, therefore, in the primary prevention of the symptoms mentioned in the ICU doctors and nurses, since there are no studies of these characteristics in our country.

Finally, it is important to point out that when primary prevention is carried out in the professional affected by the ICU, the patient who receives care also benefits, since both are part of the same sys- tem, in which the lack of health of one can affect in the quality of the other's assistance. The process cannot be understood as separate units but rather as parts of the system, with which the attitude of one of the actors influences the rest.

\section{Methodology}

A non-experimental cross-sectional correlational study was conducted [29], in order to analyze and know the association between both variables. For data collection, a non-probabilistic sampling was used using two different methodologies: a first sampling 'of convenience' surveying the responses of health professionals who attended a faceto-face congress and who voluntarily agreed to participate in the study, was then completed with the use of the 'Snowball' technique to reach professionals from different regions of the country who responded to the survey online. Thus, the sample was made up of 154 doctors and nurses from the ICU from different clinics and hospitals in Argentina.

These surveys were made up of:

- Two questions regarding whether the participant had had experiences of high emotional impact in early childhood and in the last 5 years, in order to consider the possibility of certain vulnerability.

- The Davidson Trauma Scale (DTS), which is a psychometric instrument that is made up of 17 items that correspond and evaluate each of the 17 symptoms belonging to criteria B, C and D collected in the DSM-IV for the diagnosis of PTSD [30] is a self-report scale, in which the subject must score two aspects of each item: the frequency of presentation and the severity using a 5 -point Likert scale ( 0 , never or zero severity, at 4 , daily or extreme severity), validated in Argentina by Villafañe A, et al. [31].

- The Resilience Scale (ER, a psychometric instrument made up of 25 items, ranging from 1: totally disagree, to 5 : totally agree. The total score is obtained by the sum of the scale scores and the theoretical values range from 25 to 175 . It is self-administered without a time limit, it can be taken individually and collectively [32]. The scale has been validated in Argentina by Rodriguez M, et al. [33].

- Socio-demographic variables: sex, age, place of residence, place of work, marital status, profession, years of service in the ICU.

\section{Results}

Through the analysis of the data, it was shown that in a sample of 154 health professionals, the group with a low level of resilience presented symptoms of STS in 36\%, while in the group with a moderate level of resilience, $31 \%$ presented symptoms of STS. Only $14 \%$ of those with a higher level of resilience showed some degree of STS (Tables 1A-C) [34].

Although the Chi-Square independence test indicates that both variables are statistically independent at the population level, the box plots that show the distribution of the STS values for each Resilience level and the calculation of the statistics show that at least for the analyzed sample, the mean values, the upper quartile and the median STS decrease as the professional's level of resilience increases (Figure $1 \&$ Table 2) [35]. 
Mental Health \& Human Resilience International Journal

\begin{tabular}{|c|c|c|c|}
\hline STS level & Frequency & Percentage & Cumulative percentage \\
\hline no trauma & 109 & 70,8 & 70,8 \\
\hline low & 27 & 17,5 & 88,3 \\
\hline moderate & 10 & 6,5 & 94,8 \\
\hline severe & 8 & 5,2 & 100 \\
\hline Total & 154 & 100 & \\
\hline
\end{tabular}

Table A

\begin{tabular}{|c|c|c|c|}
\hline Resilience level & Frequency & Percentage & Cumulative percentage \\
\hline low & 33 & 21,4 & 21,4 \\
\hline moderate & 93 & 60,4 & 81,8 \\
\hline high & 28 & 18,2 & 100 \\
\hline Total & 154 & 100 & \\
\hline
\end{tabular}

Table B

\begin{tabular}{|c|c|c|c|}
\hline \multicolumn{3}{|c|}{ Chi Square Test } & df \\
\hline Chi Square (Pearson) & value & 4 & 0,114 \\
\hline Likelihood & 7,457 & 4 & 0,037 \\
\hline Linear association & 10,191 & 1 & 0,016 \\
\hline
\end{tabular}

Table 1A-C: Levels of secondary traumatic stress and resilience.

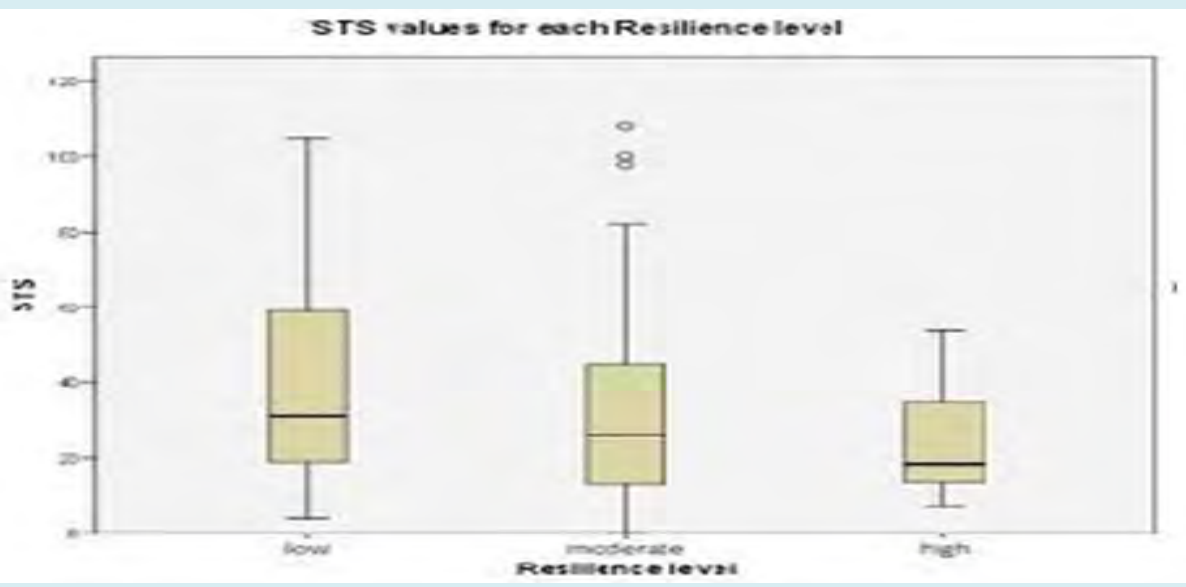

Figure 1: Distribution of secondary traumatic stress values according to resilience level.

\begin{tabular}{|c|c|c|c|c|c|}
\hline \multicolumn{7}{|c|}{ Summary STS } \\
\hline Resilience level & $\mathrm{N}$ & Media & Median & Minimum & Maximum \\
\hline low & 33 & 39,67 & 31 & 4 & 105 \\
\hline moderate & 93 & 32,17 & 26 & 0 & 108 \\
\hline high & 28 & 23,43 & 18,5 & 7 & 54 \\
\hline Total & 154 & 32,19 & 28 & 0 & 108 \\
\hline
\end{tabular}

Table 2: Case summaries. 


\section{Mental Health \& Human Resilience International Journal}

It was observed that $89.6 \%$ reported having suffered an experience of high emotional impact in their workplace, $4.5 \%$ responded negatively and $5.8 \%$ did not respond. $65.6 \%$ of the sample stated that they had not experienced a significantly negative impact event in childhood. The mean level of STS was lower for this group (28.83\%) in contrast to those who did present a negative event in childhood (40.09\%) [36].

\section{Discussion and Conclusion}

It was observed that $29.2 \%$ of the sample presented STS symptoms and $78.6 \%$ showed levels of resilience that ranged from moderate $(60.4 \%)$ to higher $(18.2 \%)$. Therefore, it can be inferred the presence of STS symptoms in ICU professionals in Argentina and that resilience acts as an important protective factor. The data corroborate the presumptive hypothesis. The importance of traumatic experiences experienced in childhood as a predisposing factor to suffer STSs was also evidenced, since the participants who declared not having traumatic impacts in childhood were less affected by STSs [37].

It means that personnel with high levels of resilience and with resilient attitudes in their work clear- ly reduce the STS, in the same way that those not affected by traumatic impacts in the first years of life, are less affected by the STS. Almost $90 \%$ of the interviewees presented a traumatic stressful situation, as in fact is expected in ICU care, but the crossover of data between the low or no emotional impact in childhood and high levels of resilience, would make it possible in the staff achieve better handling of critical situations and suffer less work stress.

However, it should be clarified that this analysis was carried out before the COVID-19 pandemic broke out, so it is expected to repeat the study for a comparison, while it could also be said that the concept of resilience has currently taken an important relevance, as a protection factor for the times that the world is having to live, so it is imperative to deepen its study.

\section{References}

1. dos Santos FD, Cunha MHF, Robazzi ML, Pedrão LJ, da Silva LA, et al. (2010) Or the stress of nurses in adult intensive care units: a literature re- view. SMAD Electronic Journal Mental Health Alcohol and Drugs 6(1): 1-21.

2. Backes MTS, Erdmann AL, Buscher A (2015) The lively, dynamic and complex environment of care in the Intensive Care Unit. Rev Lat Am Nursing 23(3): 411-418.

3. Rodrigues VM, Ferreira ADS (2011) Stressors in nurses working in Intensive Care Units. Rev Latino Am Enfermagem 19(4): 1025-1032.
4. Pearlman LA, Ian PS (1995) Vicarious Traumatization: An Empirical Study of the Effects of Trauma Work on Trauma Therapists. Professional Psychology: Research and Practice 26(6): 558-565.

5. Carpena A (2016) Empathy is possible. Emotional education for an empathic society. Hainaut 6: 48009.

6. Figley CR (2002) Treating compassion fatigue. In: Routledge B (Eds.), New York.

7. Gori SS, Hemsworth D, Chan WW, Carson A, Kazanjian A (2013) Understanding compassion satisfaction, compassion fatigue and burnout: A survey of the hospice palliative care workforce. Palliat Med 27(2): 172-178.

8. Flanigan R (2000) Vulnerability and the bioethics movement. In Bioethics Forum 16(2): 13-18.

9. Levine C (2004) The concept of vulnerability in disaster research. J Trauma Stress 17(5): 395-402.

10. Cardona OD (1993) Threat, vulnerability and risk assessment. In: Maskrey A (Eds.), Disasters are not natural, pp: 51-74.

11. Feito L (2007) Vulnerability. In Annals of the Navarra health system. Government of Navarra. Health Department 30: 7-22.

12. Diaz JSS, Callejas DF (2016) Meanings and social vulnerability to the earthquake and tsunami of February 27, 2010: the subjective dimension from the "victims". Psychological Summa UST 13(2): 23-32.

13. Ceberio MR (2021) Context and vulnerability in the covid-19 crisis: emotions and situations of the during and questions about the after. Ajayu Organ of Scientific Dissemination of the Department of Psychology UCBSP 19(1): 90-126.

14. Díaz JS (2017) Subjective dimension of vulnerability to socio-natural risks and disasters: four case studies.

15. Cyrulnik B (2013) The ugly ducklings: resilience. An unhappy childhood does not determine life. Gedisa.

16. Ceberio M (2013) Cinderellas and ugly ducklings. From devaluation to good self-esteem, Mexico, Herder

17. Martinez I, Vásquez A (2006) The resilience. The invisible resilience. Childhood, social inclusion and life tutors. Barcelona: Gedisa.

18. Moneta ME (2007) Attachment, resilience and vulnerability to illness: genotype-environment interactions. Rev GPU 3(3): 321-326. 


\section{Mental Health \& Human Resilience International Journal}

19. Méndez TP (2020) Crisis, change and vision of the future. Ludus Vitalis 28(53): 155-159.

20. Najmanovich D (2006) End of the century metamorphosis: crisis, change and complexity. Knowing the knowledge of knowledge, pp: 1-13.

21. Figley CR, Stamm BH (1999) Self-administered compassion fatigue and satisfaction questionnaire. In: Samayoa P (Eds.), El Quiché, Guatemala.

22. Pintos RAV, de Akaki CC (2000) Factors of the psychological environment in Pediatric Intensive Care Units. Mexican Journal of Pediatrics 67(2): 83-85.

23. Tyssen R, Vaglum P, Gronvold NT, Ekeberg O (2001) Suicidal ideation among medical students and young physicians: a nationwide and prospective study of prevalence and predictors. J Affect Disord 64(1): 69-79.

24. Richings JC, Khara GS, McDowell M (1986) Suicide in young doctors. Br J Psychiatry 149(4): 475-478.

25. Betta R, Morales G, Rodriguez K, Guerra C (2007) The frequency of emission of self-care behaviors and its relationship with the levels of secondary traumatic stress and depression in clinical psychologists. Psychological Thinking 3: 9-19.

26. Gentry E (2003) Attrition for empathy: the challenge of transformation. Journal of Psychotrauma for Iberoamerica 2: 4-17.

27. Salston MD, Figley Ch (2003) Secondary traumatic stress: Effects of working with survivors of criminal victimization. J Traumatic Stress 16(2): 167-174.

28. Valent P (2002) Diagnosis and Treatment of Helper Stresses, Traumas, and Illnesses. In: Figley CR (Eds.), Treating compassion fatigue, Brunner-Routledge, pp. 17-37.
29. Sampieri RH, Collado CF, Lucio PB (2010) Methodology of the investigation. Mexico DF.

30. Davidson JR, Book SW, Colket JT, Tupler LA, Roth S, et al. (1997) Assessment of a new self-rating Scale for posttraumatic stress disorder. Psychol Med 27(1): 153160.

31. Villafañe A, Milanesio MS, Marcellino CM, Amodei C (2003) The Assessment of Post-Traumatic Stress Disorder: An Approach to the Psychometric Properties of the Davidson Trauma Scale. Evaluar Magazine 3(1): 80-93

32. Wagnild GM, Young HM (1993) Development and psychometric evaluation of the Resilience Scale. J Nurs Meas 1(2): 165-178.

33. Rodriguez M, Pereyra MG, Gil E, Jofré M, Bortoli M, et al. (2009) Psychometric properties of the Argentine version of the resilience scale. Evaluar Magazine 9(1): 72-82.

34. American Pschiatric Association. (2013) Diagnostic and statistical manual of mental disorders (DSM-5). American Psychiatric Pub.

35. Vieira JM, Matos KAP, Barbosa TLA, Gomes LMX (2013) Feelings experienced by relatives of hospitalized patients in the adult intensive care center. Rev Cuba Sick pp: 1828.

36. Navarro JIV (2015) Working conditions and quality of working life in health professionals: the modulating role of resilience and self-efficacy, on burnout syndrome and engagement (Doctoral dissertation, University of Seville).

37. Figley CR (2002) Trating Compassion Fatigue, New York Brunner-R, pp: 17-37. 